\section{Prognosis of temporal lobe epilepsy in childhood}

What is the long-term outlook for children with temporal lobe epilepsy and what factors influence this prognosis? Those questions have recently been answered in a series of papers ${ }^{1-4}$ from the National Centre for Children with Seizure Disorders at the Park Hospital, Oxford-a study remarkable not only for its penetrating analyses but also for the duration and completeness of follow-up. None of the 100 children, or adults as they later became, were "lost," and those who survived-the vast majority-were reviewed in detail 13 years after the initial coding. ${ }^{5}$

The Oxford series was based on 100 consecutive children who had satisfied two physicians that they had clinical temporal lobe epilepsy and who had spikes over one or both temporal regions on electroencephalography. This was as perfect a sample of children with temporal lobe epilepsy as was possible at the time the collection was made, and neuropathological confirmation was obtained in those later treated by temporal lobectomy. Some cases of so-called benign focal epilepsy of childhood, ${ }^{6} 7$ not then recognised as an entity, may have been included, but the Oxford group is confident that their patients did not include cases of convulsive syncope or reflex anoxic seizures. ${ }^{8}$

Given, then, that a representative population of children with true temporal lobe epilepsy has been followed to adulthood, some clear prognostic factors have emerged and from them guidelines to optimum management. Of the 100 children, five died under the age of 15 . Roughly one-third of the remainder ended up as adults free of seizures and independent; another third were socially independent but receiving anticonvulsant drugs ("epileptic"); and the remainder were dependent-living as adults either with their parents or confined to institutions. The social prognosis could be predicted by factors ascertainable in childhood. There were eight adverse factors ${ }^{1}$-an intelligence quotient below 90 , onset of seizures before the age of 2 years 4 months, five or more grand mal attacks (in addition to temporal lobe epilepsy), a seizure frequency once daily or more often, a left-sided focus on electroencephalography, the hyperkinetic syndrome, epısodes of catastrophic rage, and a need for special schooling. All but one of those who became free of seizures and independent had been to normal schools.

Disorderly homes (including extreme family disruption) did not affect the outcome, but genetic factors did. Patients with a first-degree relative with febrile convulsions had a much better prognosis ${ }^{4}$; having a relative with febrile convulsions cancelled out the bad effects of the eight adverse factors. In the absence of this family history, the adult social prognosis was related to the number of adverse factors. If over three were present in childhood the adult outcome was likely to be dependence and continuing epilepsy. ${ }^{1}$

Another aspect of prognosis is gender, important in the context of marriage and parenthood. ${ }^{2}$ Girls contributed much more than boys to the next generation. Sexlessness in malesan indifference or lack of any form of sexual drive-was related to only one factor: whether or not the epilepsy had remitted before the age of 12 years. Temporal lobe epilepsy persisting during adolescence in boys was associated with lack of development of a sexual appetite.

The results of the Oxford analysis of the psychiatric prognosis were more encouraging. ${ }^{3}$ Though $85 \%$ of the children with temporal lobe epilepsy had psychiatric problems, by adult life $70 \%$ of those not severely mentally retarded were psychiatrically healthy. The $10 \%$ who developed schizophrenia-like psychoses all had left-sided temporal lobe foci. Antisocial conduct had a more complex aetiology-male sex, a focus contralateral to the preferred hand, unremittent epilepsy, low intelligence, and childhood rages proved to be a disastrous combination.

What do these findings mean to the practising doctor? Not only do they show that the adult prognosis of temporal lobe epilepsy can be predicted in childhood, but they also point the way to making the prognosis better. Firstly, there is the importance of prevention. Convulsions in the course of meningitis were the cause of many of the cases of temporal lobe epilepsy described above: seizures occurring in meningitis have long been known to worsen the prognosis, ${ }^{9}$ but prevention by adequate anticonvulsant medication before the first seizure should be practicable world wide. Long one-sided febrile convulsions cannot as yet be prevented, since most are the first in a child's life, but emergency services should be organised to allow termination of such seizures within half an hour. ${ }^{10}$

Later in childhood the question of diagnosis is paramount. Patients with convulsive syncopes, which may be confirmed by ocular compression studies, ${ }^{8}$ not surprisingly do badly with antiepileptic medication, while benign focal epilepsy of childhood (with night-time seizures in which the child makes gurgling noises, salivates, twitches on one side of the face, feels his tongue too large for his mouth, and cannot speak) always disappears by adolescence, despite numerous spikes on the electroencephalograph. ${ }^{6}$ These spikes arise from the Rolandic strip, and are often mistakenly called midtemporal. After the seizures stop these spikes go also.

During childhood the drug treatment of choice for temporal lobe epilepsy is carbamazepine ${ }^{10}$; but valproate, phenytoin, or the ketogenic diet may need a trial, and too often a combination of drugs accrues. Excessive treatment with antiepileptic drugs may actually cause seizures to persist. Whatever the frequency of the seizures the child should attend a normal school if the staff can cope.

When adolescence approaches management decisions can be made on the basis of the evidence from this Oxford study. If an adolescent has been five years without seizures, ${ }^{11}$ or has three or fewer of the Oxford adverse factors, or has a first-degree relative with febrile convulsions, then he or she should be carefully weaned off anticonvulsants, preferably under hospital supervision. If seizures persist, and particularly in a boy with more than three of the Oxford adverse factors and with no firstdegree relative with febrile convulsions, then temporal lobectomy in a centre experienced in epilepsy surgery deserves very serious consideration. Such children are not likely to remit spontaneously. If the operation is to allow them social and sexual normality without further seizures or drugs, the timing may be crucial: the age for surgery should be no later than the onset of adolescence.

\footnotetext{
${ }^{1}$ Lindsay J, Ounsted C, Richards P. Long-term outcome in children with temporal lobe seizures. I. Social outcome and childhood factors. Develop Med Child Neurol 1979;21:285-98.

${ }^{2}$ Lindsay J, Ounsted C, Richards P. Long-term outcome in children with temporal lobe seizures. II. Marriage, parenthood and sexual indifference. Develop Med Child Neurol 1979;21 :433-40.

${ }^{3}$ Lindsay J, Ounsted C, Richards P. Long-term outcome in children with temporal lobe seizures. III. Psychiatric aspects in childhood and adult life. Develop Med Child _Neurol 1979;21:630-6.

${ }^{4}$ Lindsay J, Ounsted C, Richards P. Long-term outcome in children with temporal lobe seizures. IV. Genetic factors, febrile convulsions, and the remission of seizures. Develop Med Child Neurol (in press).
} 
5 Ounsted C, Lindsay J, Norman R. Biological factors in temporal lobe epilepsy. London: Heinemann, 1966.

${ }^{6}$ Anonymous. Benign focal epilepsy of childhood. Br Med f 1975 ;iii:451-2.

7 Beaussert M, Faou R. Evolution of epilepsy with Rolandic paroxysmal foci : a study of 324 cases. Epilepsia $1978 ; 19: 337-42$

${ }^{8}$ Stephenson JBP. Reflex anoxic seizures ("White breath-holding"): nonepileptic vagal attacks. Arch Dis Childh 1978;53:193-200.

9 Ounsted C. Significance of convulsions in children with purulent meningitis. Lancet 1951 ; i :1245-8.

${ }^{10}$ O'Donohoe NV. Epilepsies of childhood. London: Butterworths, 1979.

11 Annegers JF, Hauser WA, Elveback LR. Remission of seizures and relapse in patients with epilepsy. Epilepsia 1979;20:729-37.

\section{Corticosteroids and hypothalamic-pituitary- adrenocortical function}

When corticosteroids are used for suppressing diseases such as asthma, ulcerative colitis, chronic active hepatitis, and systemic lupus erythematosus high doses may be given for prolonged periods. The inhibition of hypothalamic-pituitaryadrenocortical (HPA) function induced by these exogenous glucocorticoids may persist for six to 12 months after treatment is withdrawn. ${ }^{1}$ This inhibition may cause acute adrenal insufficiency with circulatory collapse in the presence of stress. $^{2}$

Suppression of secretion of adrenocorticotrophic hormone (ACTH) and atrophy of the adrenal cortex become progressively more definite as the doses of steroids exceed physiological levels (more than $7.5 \mathrm{mg}$ /day prednisolone ${ }^{3}$ ) and the treatment is prolonged. ${ }^{134}$ The suppression is less when the steroid is given as a single dose in the morning, matching the circadian pattern, than when a single evening dose is given or the dose is divided through the day. ${ }^{5}{ }^{6}$ Furthermore, if the morning dose is given on alternate days or even less frequently less inhibition results than from daily dosing even when the total amount of drug given is identical. ${ }^{14}$ An alternate-day regimen may not, however, maintain therapeutic control, particularly with diseases such as asthma. ${ }^{17}$ Injections of ACTH are less likely to cause suppression of $\mathrm{HPA}^{1}$ but are less effective and more difficult to administer. Giving ACTH concurrently with steroids ${ }^{8}$ or transferring from oral steroids to ACTH does not speed recovery from HPA inhibition. ${ }^{9}$

In general, therefore, the minimum possible dose of steroid should be given for the minimum period of time-a requirement that may conflict with the sort of regimen needed to control a chronic disease. Steroids should not be given more often than once daily, in the morning. In patients taking high enough doses of steroids to suppress the adrenal glands the occurrence of any form of stress is an indication for an increase in dose, and patients treated with steroids within the previous two to three months should be restarted on treatment. When the interval since steroid treatment is three to 12 months resumption of treatment should depend on clinical assessment of signs of adrenal insufficiency.

How patients should be weaned from suppressive steroid treatment depends on the underlying disease and on the duration of treatment. ${ }^{10}$ If the disease has resolved and the duration was only weeks or months, the steroids may be reduced quickly-for example, by $2.5-5 \mathrm{mg}$ prednisolone a day every two or three days. ${ }^{11}$ If resolution is uncertain or treatment has lasted for years, withdrawal should be slowerfor example, by $2.5 \mathrm{mg}$ every one to three weeks ${ }^{11}$; a disease marker-for example, the sedimentation rate-may indicate occasionally that withdrawal should be even slower. Once the dose of prednisolone has been reduced to $10 \mathrm{mg}$ a day smaller decrements should be made with $1 \mathrm{mg}$ tablets. The integrity of HPA function may be tested by measuring morning plasma cortisol concentrations or by the Synacthen test. ${ }^{12}$ The response to insulin-induced hypoglycaemia ${ }^{13}$ will indicate whether response to stress has recovered, and this may be important for two to three months after withdrawal.

Short courses of high doses of steroids are used in treating septic shock, various forms of malignant disease, severe skin disorders, and cerebral oedema. In these circumstances treatment with steroids is commonly stopped abruptly in the belief that adrenal function is uncompromised. In fact, short-term administration of steroids does produce abnormalities of HPA function, sometimes within 24 hours. These include reduction in basal cortisol concentrations, ${ }^{56}$ loss of diurnal variation, ${ }^{6}$ a diminished response by the adrenals to exogenous $\mathrm{ACTH},{ }^{14}$ and blunted or absent responses to insulin-induced hypoglycaemia. ${ }^{15-17}$ Two days after the administration of prednisone $25 \mathrm{mg}$ twice daily for five days, the cortisol responses to both hypoglycaemia and synthetic ACTH are reduced by about half; some decrease in response $(18 \%)$ to the latter stimulus is still present five days after the treatment. ${ }^{18}$

Clearly, then, steroids may affect HPA function within a few days of starting treatment, even though the effects usually disappear rapidly once treatment is discontinued. Here again once-daily prescribing, where possible, may be least harmful. Many patients treated with steroids will be under continuing stress when the drugs are stopped, and a 50\% reduction in the adrenal glands' ability to respond to this stress even for a few days may be important-particularly since deterioration in the patient's condition may be attributed wrongly to the disease. In these circumstances, withdrawing steroids gradually over five to seven days is preferable to abrupt discontinuation. In addition, if the patient's condition suggests the possibility of adrenal insufficiency after a shortterm course of steroids, the integrity of HPA function should be tested by the response to hypoglycaemia or the Synacthen test or, when this is clinically inappropriate, treatment with steroids should be reintroduced.

Suppressive treatment with steroids is being used frequently in both long-term and short-term courses. Generally the inherent dangers are recognised but prescribing in and control of individual cases may fall short of the ideal. Accurate information about treatment with steroids, past or present, is often difficult to obtain when it is most needed-immediately in the presence of acute illness. A high standard of care and of record keeping is needed if patients are to obtain all the benefits of treatment with steroids with as few of the disadvantages as possible.

1 Axelrod L. Glucocorticoid therapy. Medicine (Baltimore) 1976;55:39-65.

2 Salassa RM, Bennett WA, Keating Jr FR, Sprague RG. Postoperative adrenal cortical insufficiency occurrence in patients previously treated with cortisone. $\mathcal{F A M A}$ 1953;152:1509-1515.

${ }^{3}$ Livanou T, Ferriman D, James V. Recovery of hypothalamo-pituitaryadrenal function after corticosteroid therapy. Lancet 1967;ii :856-859.

4 Melby JC. Systemic corticosteroid therapy: pharmacology and endocrinologic considerations. Ann Intern Med 1974;81:505-512.

5 Grant S, Forsham P, Diraimondo V. Suppression of 17-hydroxycorticosteroids in plasma and urine by single and divided doses of triamcinoline. $N$ Engl f Med $1965 ; 273: 1115-8$.

${ }^{6}$ Nichols T, Nugent C, Tyler F. Diurnal variation in suppression of adrenal function by glucocorticoids. $\mathcal{F}$ Clin Endocrinol Metab 1965 ;25 : 343-349.

${ }^{7}$ Fauci AS. Alternate-day corticosteroid therapy. Am $\mathcal{f}$ Med 1978;64: 729-731. 